Tabelle IV

(Versuchsstation Speyer).

\begin{tabular}{|c|c|c|c|c|c|c|c|c|c|}
\hline 26. V. 1914 & $1 \mathrm{a}$ & $1 b$ & $2 a$ & $2 \mathrm{~b}$ & 3 & $4 a$ & $4 b$ & 5 & 6 \\
\hline Spezifisches Gewicht & 1,0038 & 1,0082 & 1,0035 & 1,0032 & 0,9962 & 0.9958 & 0,9959 & 0,9961 & 0,9942 \\
\hline Alkohol. . . . & 3,69 & 3.58 & 3.69 & 3,69 & 7,26 & 7,26 & 7,26 & 7,73 & 8,07 \\
\hline Extrakt. . . . & 2.48 & 2.40 & 2,44 & 2,34 & 2,21 & 2,20 & 2,25 & 2,20 & 1,97 \\
\hline Asche . . . . & 0,275 & 0,262 & 0,220 & 0,240 & 0,18 & 0.21 & 0,21 & 0,21 & 0,16 \\
\hline Titrierbare Säure . & 1,07 & 0,98 & 1,00 & 0,84 & 0,79 & 0,77 & 0,75 & 0,69 & 0,74 \\
\hline Flithtige Sâure. . & 0,10 & 0,06 & 0,06 & 0,05 & 0,04 & 0,04 & 0,04 & 0,05 & 0,04 \\
\hline Nichtflüchtige Säure & 0,94 & 0,85 & 0,92 & 0,78 & 0,74 & 0,72 & 0,70 & 0,63 & 0,69 \\
\hline Milchsäure . . & 0,71 & 0,71 & 0,75 & 0,72 & 0,51 & 0.55 & 0,55 & 0,51 & 0,42 \\
\hline Zucker . . & 0,10 & 0,10 & 0,10 & 0,10 & - & - & - & - & - \\
\hline Polarisation . & - & - & - & - & \pm 0 & \pm 0 & \pm 0 & \pm 0 & \pm 0 \\
\hline Alkalinat der Asche & 1,70 & 2,30 & 1,90 & 2,20 & 1,50 & 2,00 & 1,85 & 1,85 & 1,50 \\
\hline Gesamt.Weinsäure. & 0,82 & 0,29 & 0,35 & 0,25 & 0,28 & 0,20 & 0,20 & 0,16 & 0,28 \\
\hline Freie Weinsäure. & 0,07 & 0 & 0,06 & 0 & 0,06 & 0,09 & 0,09 & 0 & 0,05 \\
\hline Weinstein. . . & 0,32 & 0,36 & 0,36 & 0,31 & 0.28 & 0.25 & 0.26 & 0,20 & 0,28 \\
\hline Extraktrest I . & 1,54 & 1,55 & 1,52 & 1,56 & 1,47 & 1,48 & 1,55 & 1,57 & 1,28 \\
\hline$\pi \quad$ II . & 1,41 & 1,47 & 1,44 & 1,50 & 1,42 & 1,41 & 1,50 & 1,51 & 1,23 \\
\hline Săurerest. & 0,74 & 0,70 & 0,71 & 0,65 & 0,60 & 0,62 & 0,60 & 0,55 & 0,55 \\
\hline Glycerin . . . & 0,42 & 0,39 & 0,38 & 0,38 & 0,56 & 0,61 & - & 0,58 & 0,57 \\
\hline Alkobol: Glycerin & & & & & & & & & \\
\hline$=100 \mathrm{a}$ & 11,4 & 10,9 & 10,3 & 10,3 & 7,7 & 8,4 & - & 7,5 & 7,1 \\
\hline Verhalten gegen & & & & & & & & & \\
\hline Alkohol. . & \multicolumn{6}{|c|}{ normal } & - & \multicolumn{2}{|c|}{ normal } \\
\hline Silbernilrat. . & \multicolumn{4}{|c|}{ normal } & & & - & \multicolumn{2}{|c|}{, } \\
\hline Chlorbarium . & \multirow{2}{*}{\multicolumn{4}{|c|}{ stärker }} & \multirow{3}{*}{\multicolumn{2}{|c|}{ normal }} & - & \multicolumn{2}{|c|}{$\pi$} \\
\hline Beschaff-nheit des & & & & & & & & & \\
\hline Extraktes . . & & ht & 60 & & & mal & - & & \\
\hline
\end{tabular}

\title{
Die Weinernte 1917 in der Pfalz.
}

Von

Otto Krug und Hans Filehner.

Mitteilung der Landwirtschaftlichen Kreisversuchsstation Speyer.

[Ërgegangen an 4. Dezember 1917.]

Ein Weinjahr harter Arbeit aber auch reich an ungeahnten Erfolgen liegt wieder hinter uns. Der Kriegsherbst 1917 hat uns einen Wein beschert, der zu den besten der letzten Jahrzehnte zäblen wird, der aber nicht minder auch zu einem wahren „Sorgenbrecher" aus allen den wirtschaftlichen Nöten für unsere Winzer geworden ist, in die sie ohne ihre Schuld durch die vielen Mibjahre geraten waren. Der Jahrgang 1917 wird daher in der Geschichte des pfälzischen Weinbaues einen Markstein bilden 
und noch in späteren. Tagen werden unsere Winzer Ursache haben, dieses Kriegsherbstes rübmend $\mathrm{zu}$ gedenken.

Das Rebholz hatte dank der milden Spätjahrswitterung im allgemeinen eine befriedigende Reife erlangt, so daß es auch befähigt war, der starken und lang andauernden Winterkälte zu widerstehen. Nur vereinzelt machten sich später Frostschäden dadurch bemerkbar, daß namentlich in den von Hagel und Krankheiten beschädigten Weinbergen manche Fruchtaugen schwarz geworden waren und vielfach auch Rebschenkel, besonders in den alten Gutedel-Wingerten des oberen Gébirges, stark aufgerissen waren.

Durch den überaus strengen und lang andauernden Winter, der seine Herrschaft fast bis in den Monat Mai geltend machte, erlitten die Reb- und Bodenarbeiten eine große Verzögerung. Bei der herrschenden Leutenot galt es daher alle Kräfte zưsammen zu nehmen, um die Arbeiten noch rechtzeitig vor Eintritt des Frübjabrs zu. beendigen. Trotz des ungünstigen und rauhen Wetters sah man daher während der wärmeren Tagesstunden neben Kriegsgefangenen und älteren Winzern meist Frauen und Mädchen eifrig die Arbeiten des Schneidens, Bindens und Biegens verrichten. Ueberall spürte man schon deutlich den Einfluf der hohen Weinpreise und das Bestreben, nichts zur Sicherung einer guten Weinernte zu versäumen.

Bei dem Mangel an Arbeitskräften und der schwierigen und kostspieligen Beschaffung von Stallmist und Kunstdünger konnte leider in vielen Weinbergen eine gründliche Düngung und Bodenbearbeitung nicht vorgenommen werden. Leiztere wäre um so notwendiger gewesen, als die Weinberge aus dem Vorjahre her schon vielfach sehr stark verunkrautet waren und es bei der mangelhaften Düngung jetzt mebr als je galt, die natürlichen Nährstoffe des Bodens flüssig zu machen und die Wurzeln zu lebhaftem Wachstum anzuregen. Das ungünstige und kalte Wetter im Monat April brachte eine wesentliche Verzögerung in der Entwickelung der Reben. Man sah dies wwar in Winzerkreisen im Hinblick auf die verminderte Gefahr von Frostschäden nicht ungern. Auch herrschte vielfach die Ansicht, daß dem strengen Winter auch die hauptsächlichsten tierischen Schädlinge des Weinbaues zum Opfer gefallen seien, eine Hoffnung, die sich leider bald als trügerisch erweisen sollte. Der Monat Mai brachte endlich so sommerlich warme Tage, daß der Austrieb der Reben ungemein rasch erfolgte. Bald standen die Weinberge in herrlichem Grün und auch die Zahl der Gescheine, namentlich in den gut gepflegten Weinbergen berechtigte zu den besten Hoffnungen.

Leider war bald in vielen Lagen, namentlich des oberen und mittleren Gebirges, ein massenhaftes Auftreten des Rebstichers zu beobachten. Trotz teilweise eifriger Bekämpfung durch Einsammeln der Käfer und Wickel fielen der Gefräligkeit des Insektes doch zahlreiche Gescheine zum Opfer. Am 18. Mai wurden leider wiederum verschiedene Gemeinden des oberen Gebirges von einem Hagelwetter von ganz auberordentlicher Stärke heimgesucht, und es wurden namentlich die Gemeinden Maikammer, Alsterweiler, Edenkoben, Edesheim, St. Martin, Diedesfeld, Hambach sehr schwer betroffen. In der Gemeinde Maikammer, die im Mittelpunkte des Unwetters lag, wurden etwa $90 \%$ der Ernte vernichtet und man kann den Schaden dieser Gemeinde bei Berücksichtigung der späteren Herbstpreise auf etwa 8 Millionen Mark veranschlagen. Durch die weiter herrschende sommerlich warme Witterung und die zahlreichen Gewitterregen machten die Reben dann sehr schnelle Fortschritte, Die Blüte, die fast überall im ersten Drittel des Monats Juni erfolgte, nahm eỉnen üheraus günstigen 
und raschen Verlauf. Diesem Umstand ist es auch zu danken, daß der Heuwurm, der nur in manchen Lagen stärker auftrat, besonders großen Schaden nicht anzurichten vermochte.

Dagegen konnte schon Ende Mai in verschiedenen Lagen ein starker Befall von Peronospora festgestellt werden. Die feuchtwarme Witterung mit teilweiser starker Taubildung begünstigte die weitere Entwickelung dieser gefürchteten Rebkrankheit auBerordentlich. Leider waren unsere Winzer durch die verspätete Lieferung von Kupfervitriol meist nicht in der Lage, rechtzeitig den Kampf gegen die Blattfallkrankheit aufzunehmen und war dies nur denjenigen möglich, die das Glück hatten, vom Vorjahre her noch etwas Kupfervitriol zu besitzen. Der Schaden, der hierdurh entstanden ist, muß als ein recht erheblicher bezeichnet werden, indem der Pilz dadurch Zeit gewann, auch die jungen Träubchen zu überziehen und hier Lederbeeren zu erzeugen. Einer weiteren Ausbreitung der Peronospora konnte glücklicherweise durch die häufige und sachyemäße Kupferung Einhalt geboten werden. Wenn trotzdem in manchen Lagen diesen Maßnahmen der Erfolg versagt blieb, so dürfte die Ursache hierfür in erster. Linie auf die unrichtige Ausführung des Spritzens durch meist ungeschulte und unerfahrene Hilfskräfte zuräckzuführen sein. Bei dem Mangel an Kupfervitriol sahen sich die Winzer vielfach genötigt, bei dem späteren Spritzen an Stelle der bewährten Bordelaiserbrühe das Perozid zu verwenden. Wenn dieses Mittel sich auch im allgemeinen rccht gut bewährt bat, so stehen unsere Winzer doch diesem neuen Bekämpfungsmittel begreiflicherweise noch etwas skeptisch gegenüber. Am 30. Juli ging leider in der Umgebung von Grünstadt ein schweres Hagelwetter nieder, das in den davon betroffenen Weinbergen nicht unerheblichen Schaden anrichtete.

Trotż der feuchten und wenig warmen Witterung im August machte die Reife der Trauben weiter solche Fortschritte, daß mit einer sehr frühen Lese gerechnet werden konnte.

Der Stand der Weinberge war zu Anfang September je nach der Behandlung und Pflege ein außerordentlich verechiedener. Man traf ganze Gemarkungen mit durchweg schönem und gesundem Bthang, daneben aber auch Lagen, in denen Blätter und Truben fast völlig der Peronospora zum Opfer gefallen waren. Das warme und schöne Septemberwetter förderte nicht nur die Qualität der Trauben außerordentich, sondern hielt auch eine drohende Fäulnis fast völlig zurück. Schon am 1('. September konnte mit der Lese der Portugieser begonnen werden, die fast durchweg von selten schöner und gesunder Beschaffenheit waren. Nach Beendigung des Portugieserherbstes folgte in der letzten Septemberwoche und Anfangs Oktober nuch allgemein die Lese der Weißweine, die von bestem Herbstwetter begünstigt war. Wie vorauszusehen war, schwankten die Herbsterträge ganz außerordentlich; während viele Winzer das Glück hatten, nahezu einen vollen Herbst zu machen, mußten sich andere wiederum mit einem sehr bescheidenen Herbst begnügen. Neben den Portugiesertrauben standen wohl die Traminer am schönsten, dagegen hatten die Rieslingtrauben durch die Peronospora, die Lederbeerenkrankheit und teilweise auch durch Wurmschaden den größten Abgang aufzuweisen. Die Beschaffenheit der geberbsteten Moste war durehweg eine vorzügliche und auch in den geringeten Lagen wurden fast durchweg hohe Mostgewichte bei niedrigem Säuregehalt festgestellt. Der Jahrgang' 1917 kann sich daher in bezug auf seine Bescbaffenheit den Jahrgängen 1911 und 1915 ebenbürtig zur Seite-stellen, wenn er auch in bezug auf seine "Spitzen" den Jahrgang 1915 nicht erreichen dürfte. 
Bei der allgemeinen Weinknappheit und bei dem fortwährend großen Heeresbedarf konnte es nicht fehlen, daß auch die Mostpreise eine ganz ungeahnte Höhe erreichten und daß die Preise gegenüber dem Vorjahr noch eine weitere Steigerung um das 2- bis 3-fache erfubren. Je nach Lage und Güte betrug der Preis für die Logel (40 Liter) Trebermost bei Rotwein (Portugieser) etwa 100 bis 130 Mark und bei Weißmost etwa 90 bis 160 Mark. Wenn sich auch angesichts dieser fast märchenhaften Preise der Weinhandel anfänglich begreiflicherweise eine gewisse Zurückhaltung auferlegte, so fand doch der "Neue" wegen seiner hervorragenden Güte bald willige Abnehmer. Trotz dieser günstigen Verhältnisse haben manche Winzer in Erwartung weiterer Preissteigerungen sogar noch mit dem Verkauf zurückgehalten. Nach den vielen Jabren getäuschter Hoffnungen hat das Kriegsjahr 1917 unsere Winzer für die aufgewendete Sorge und Mühe in reichstem Maße entschädigt und wenn der Volksmund schon jetzt den 1917-er als „Hypothekentilger“ bezeichnet, so liegt hierin ein treffender Hinweis auf die hohen Gewinne, die die Winzer in diesem Jahre erzielt haben.

Die Zahl der untersuchten Mostproben betrug 362. Von diesen entfallen:

$$
283 \text { Proben auf Weißmost, und }
$$

79 " "Rotmost,

die sich auf die einzelnen Weinbaugebiete der Pfalz, wie folgt, verteilen:

Tabelle I. Art und Zahl der untersuchten Moste.

\begin{tabular}{|c|c|c|c|c|c|c|c|c|c|c|c|c|}
\hline \multirow{2}{*}{$\begin{array}{c}\text { Art } \\
\text { des } \\
\text { Mostes }\end{array}$} & \multicolumn{2}{|c|}{$\begin{array}{l}\text { Ober- } \\
\text { Haardt }\end{array}$} & \multicolumn{2}{|c|}{$\begin{array}{l}\text { Mittel- } \\
\text { Haardt }\end{array}$} & \multicolumn{2}{|c|}{$\begin{array}{l}\text { Unter- } \\
\text { Haardt }\end{array}$} & \multicolumn{2}{|c|}{$\begin{array}{c}\text { Alseuz-, Eis } \\
\text { und Glantal } \\
\text { (Nordpfalz) }\end{array}$} & \multicolumn{2}{|c|}{$\begin{array}{l}\text { Öbrige Pfalz } \\
\text { (Rheinebene) }\end{array}$} & \multicolumn{2}{|c|}{ Im ganzen } \\
\hline & $\begin{array}{l}\text { Zahl der } \\
\text { Proben }\end{array}$ & $\%$ & $\begin{array}{l}\text { Zahl der } \\
\text { Proben }\end{array}$ & $\%$ & $\mid \begin{array}{c}\text { Zahl der } \\
\text { Proben }\end{array}$ & $\%$ & $\begin{array}{l}\text { Zahl der } \\
\text { Proben }\end{array}$ & $\%$ & $\begin{array}{l}\text { Zahl der } \\
\text { Proben }\end{array}$ & $\%$ & $\begin{array}{c}\text { Zabl der } \\
\text { Prol en }\end{array}$ & $\%$ \\
\hline Rotmost & 29 & 7.6 & 27 & 7,0 & 17 & 4,4 & 3 & 0.7 & 3 & 0,7 & 79 & 20,4 \\
\hline Weifmost & 96 & 25.8 & 57 & 15.2 & 52 & 13,8 & 69 & 18.5 & 9 & 6.3 & 283 & 79.6 \\
\hline Zusammen: & 125 & $|33,4|$ & 84 & $|22,2|$ & 69 & $\mid 18,2$ & 72 & $|19,2|$ & 12 & 7,0 & 362 & 100,0 \\
\hline
\end{tabular}

Die ermittelten Mostgewichte und Säuregehalte sind aus den Tabellen II und III $z u$ ersehen.

Tabelle II. Mostgewichte.

a) Rotmoste.

\begin{tabular}{|c|c|c|c|c|c|c|c|c|c|c|c|c|}
\hline \multirow{2}{*}{$\begin{array}{l}\text { Most- } \\
\text { gewichte } \\
\text { in } \\
\text { Oechsle. } \\
\text { Graden }\end{array}$} & \multicolumn{2}{|c|}{$\begin{array}{l}\text { Ober- } \\
\text { Haardt }\end{array}$} & \multicolumn{2}{|c|}{$\begin{array}{l}\text { Mittel- } \\
\text { Haardt }\end{array}$} & \multicolumn{2}{|c|}{$\begin{array}{l}\text { Unter- } \\
\text { Haardt }\end{array}$} & \multicolumn{2}{|c|}{$\begin{array}{l}\text { Alsenz-, Eis- } \\
\text { und Glantal } \\
\text { (Nordpfalz) }\end{array}$} & \multicolumn{2}{|c|}{$\begin{array}{l}\text { Übrige Pfalz } \\
\text { (Rheinebene) }\end{array}$} & \multicolumn{2}{|c|}{ Im ganzen } \\
\hline & $\mid \begin{array}{r}\text { Zahl der } \\
\text { Proben }\end{array}$ & $\%$ & $\begin{array}{l}\text { Zahl der } \\
\text { Proben }\end{array}$ & $\%$ & $\begin{array}{l}\text { Zahl der } \\
\text { Proben }\end{array}$ & $\%$ & $\begin{array}{l}\text { Zahl der } \\
\text { Proben }\end{array}$ & $\%$ & $\begin{array}{l}\text { Zahl der } \\
\text { Prohen }\end{array}$ & $\%$ & $\begin{array}{l}\text { Zahl der } \\
\text { Proben }\end{array}$ & $\%$ \\
\hline 50 & 1 & 1 & - & - & 2 & 2.6 & - & - & 1 & 1,3 & 4 & 5,2 \\
\hline 6 & 11 & 14 & 3 & 3,9 & 6 & 7,6 & 1 & 1,3 & 1 & 1,3 & 22 & 28,1 \\
\hline & 14 & 17 & 15 & 18,9 & 8 & 10,0 & 1 & 1, & 1 & 1,3 & 3 & 48,9 \\
\hline 80 & 3 & 3,9 & 9 & 11,3 & - & - & 1 & 1,3 & - & - & 13 & 16,5 \\
\hline $90-99.9$ & - & - & $\ldots$ & - & 1 & 1,0 & - & $1-$ & 一 & 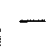 & 1 & 1,3 \\
\hline $\mathrm{mI}$ & $2 y$ & 36,6 & $\angle 7$ & 34,1 & 17 & 21,5 & 3 & 3,9 & 3 & 3,9 & 79 & 1160,0 \\
\hline
\end{tabular}




\begin{tabular}{|c|c|c|c|c|c|c|c|c|c|c|c|c|}
\hline \multirow{2}{*}{$\begin{array}{l}\text { Most. } \\
\text { gewichte } \\
\text { in } \\
\text { Oechsle- } \\
\text { Graden }\end{array}$} & \multicolumn{2}{|c|}{$\begin{array}{l}\text { Ober- } \\
\text { Haardt }\end{array}$} & \multicolumn{2}{|c|}{$\begin{array}{l}\text { Mittel- } \\
\text { Haardt }\end{array}$} & \multicolumn{2}{|c|}{$\begin{array}{l}\text { Unter- } \\
\text { Haardt }\end{array}$} & \multicolumn{2}{|c|}{$\begin{array}{l}\text { Alsenz-, Eis- } \\
\text { und Glantal } \\
\text { (Nordpfalz) }\end{array}$} & \multicolumn{2}{|c|}{$\begin{array}{l}\text { Ubrige } \Gamma \text { falz } \\
\text { (Rheinebene) }\end{array}$} & \multicolumn{2}{|c|}{ Im ganzen } \\
\hline & $\begin{array}{l}\text { Zahl der } \\
\text { Proben }\end{array}$ & $\%$ & $\begin{array}{l}\text { Zahl der } \\
\text { Proben }\end{array}$ & $\%$ & $\begin{array}{l}\text { Zahlder } \\
\text { Proben }\end{array}$ & $\%$ & $\begin{array}{l}\text { Zahl der } \\
\text { Proben }\end{array}$ & $\%$ & $\begin{array}{c}\text { Zahl der } \\
\text { Proben }\end{array}$ & $\%$ & $\begin{array}{l}\text { Zablider } \\
\text { Proben }\end{array}$ & $\%$ \\
\hline
\end{tabular}

b) Weißmoste.

\begin{tabular}{r|r|r|r|r|r|r|r|r|r|r|r|r}
$50-59,9$ & 2 & 0,8 & - & & - & - & 1 & 0,4 & - & - & 3 & 1,2 \\
$60-69,9$ & 15 & 4,9 & - & - & 2 & 0,8 & - & - & 1 & 0.4 & 18 & 6,1 \\
$70-79,9$ & 62 & 21,1 & 1 & 0,4 & 7 & 2,8 & 10 & 4,0 & 5 & 2,0 & 85 & 50,3 \\
$80-89.9$ & 17 & 5,6 & 9 & 3,6 & 21 & 7,0 & 45 & 156 & 3 & 1,2 & 95 & 33,0 \\
$90-99,9$ & - & - & 30 & 12,0 & 21 & 7,0 & 13 & 4,4 & - & - & 64 & 23,4 \\
$100-109.9$ & - & - & 17 & 5,6 & 1 & 0,4 & - & - & - & -- & 18 & 6,0 \\
\hline Zusammen: & 96 & 32,4 & 57 & 21,6 & 52 & $\mid 18,0$ & 69 & $\mid 24,4$ & 9 & 3,6 & 283 & 100,0
\end{tabular}

Tabelle III. Freie Säuren.

a). Rutmosie.

\begin{tabular}{c|r|r|r|r|r|r|r|r|r|r|r|r|r}
$\begin{array}{c}\text { Freie Säuren } \\
\mathbf{g} \text { in } 100 \mathrm{ccm} \\
0,60-0,79\end{array}$ & - & - & 1 & 1,3 & - & - & 2 & 2,6 & - & - & 3 & 3,9 \\
$0,810-0,99$ & 6 & 7.6 & 16 & 20.1 & 8 & 10,0 & 1 & 1,3 & - & - & 31 & 39,0 \\
$1,00-1.19$ & 17 & 21,5 & 10 & 13,0 & 7 & 8,4 & - & - & 1 & 1.3 & 35 & 44.2 \\
$1,20-1,39$ & 5 & 6.4 & - & - & 1 & 1,3 & - & - & 1 & 1,3 & 7 & 9,0 \\
$1,40-1,59$ & 1 & 1,3 & - & - & 1 & 1,3 & - & - & - & - & 2 & 2,6 \\
$1,60-1,79$ & - & - & - & - & - & - & - & - & 1 & 1.3 & 1 & 1,3 \\
\hline Zusammen: & 29 & 36,6 & 27 & 34,4 & 17 & 21,0 & 3 & 3,9 & 3 & 3,9 & 79 & 100,0
\end{tabular}

b) Weißmoste.

\begin{tabular}{r|r|r|r|r|r|r|r|r|r|r|r|r|} 
unter 0,60 & 1 & 0,4 & - & - & - & - & 2 & 0,8 & - & - & 3 & 1,2 \\
$0,60-0,79$ & - & - & 28 & 94 & 20 & 8.0 & 43 & 14.9 & - & - & 91 & 32,3 \\
$0,80-0.99$ & 16 & 5,3 & 26 & 9,0 & 26 & 9,0 & 23 & 7,9 & - & - & 91 & 31,2 \\
$1,00-1,19$ & 46 & 15,9 & 3 & 1,2 & 2 & $0, \times$ & 1 & 0,4 & 2 & 0,8 & 54 & 19,1 \\
$1,20-1,39$ & 26 & 9,0 & - & - & 4 & 1,6 & - & - & 4 & 1,6 & 34 & 12,2 \\
$1,40-1,59$ & 5 & 20 & - & - & - & - & - & - & 2 & 0,8 & 7 & 2,8 \\
$1,60-1,79$ & 2 & 0,8 & - & - & - & - & - & - & - & - & 2 & 0,8 \\
$1,80-1,99$ & - & - & - & - & - & - & - & - & 1 & 0,4 & 1 & 0,4 \\
\hline Zusammen: & 96 & 33,4 & 57 & 19,6 & 52 & 19,4 & 69 & 24,0 & 9 & 3,6 & 283 & 100,0
\end{tabular}

Bei den Rotmosten ist hiernach das durchschnittliche Mostgewicht für die Ober-Haardt 70,70, für die Mittel-Haardt $76,7^{\circ}$, für die Unter.Haardt und das Zellertal $71,6^{\circ}$, für das Alsenz-, Eis- und Glantal $72,7^{\circ}$ und für die übrige Pfalz (Rheinebene) $67,0^{\circ}$. Der durchschnittliche Säuregehalt ist $10,9 \%$ bezw. $9,6 \% 00,10,3 \%$, $8,2 \%$ und $13,6 \%$. Das höchste Mostgewicht beträgt $95,6^{\circ}$ bei $8,25 \%$ Säure (Unter-Haardt), das niedrigste $57,3^{\circ}$ bei $14,4 \%$ Säure.

Der höchste Säuregehalt ist $17,7 \%$ bei $\tilde{0} 9,8^{\circ}$ Oechsle (Rheinebene). Letztere Zahlen wurden bei einem aus der Kiliansrebe aus der Gemarkung Dudenhofen gewonnenen Traubensaft festgestellt und es sind diese Befunde wieder ein Beweis dafür, 
daß auch im Jahre der Reife aus dieser Traubensorte ohne Verbesserung bezw. Streckung mit Zuckerwasser ein konsumfähiger Wein nicht erzielt werden kann. Der niedrigste Säuregehalt betrug $7,1 \%$ bei $81,1^{\circ}$ Oechsle (Glantal).

Bei den Weißmosten berechnet sich das durchschnittliche Mostgewicht für die Oberhaardt zu $74,4^{\circ}$, für die Mittelhaardt zu 96,0 , für die Unterhaardt und das Zellertal zu 87,\%0, für das Alsenz-, Eis- und Glantal zu 84,9 $9^{\circ}$ und für die übrige Pfalz (Rheinebene) zu $75,3^{0}$, der durchschnittliche Säuregehalt zu 11,5\% bezw. $7,9 \%$, $8,7 \% 00,7,5 \%$ und $13,5 \%$. Das höchste Mostgewicht weist die Mittelhaardt mit $109,0^{\circ}$ Oechsle bei einem Säuregehalt von $6,2^{\%} / 00$ auf, das niedrigste die Oberhaardt mit $53,3^{0}$ bei $13,2 \%$ Säure. Der höchste Säuregehalt ist $18,0 \%$ bei einem Mostgewicht von $66,8^{\circ}$ (Rheinebene, Gemarkung Heiligenstein), der niedrigste $5,6 \%$ bei einem Mostgewicht von $76^{\circ}$ (Alsenztal).

Für das Weinbaugebiet der Pfalz sind daher in diesem Jahre die gesetzlichen Voraussetzungen für die Zulässigkeit einer Zuckerung im allgemeinen nicht gegeben.

Die Gärung der Moste verlief sehr rasch und vollständig und haben sich die Jungweine bis jetzt zu sehr sauberen, kräftigen und rassigen Gewächsen entwickelt. Angesichts der niedrigen Säure empfiehlt es sich, in diesem Jahre ganż besonders die Jungweine früher als sonst von der Hefe abzulassen, da sonst der stahlige und rassige Charakter der Weine, der wesentlich auf der Art ihrer Säure beruht, notleiden könnte. Da ferner manche Weine starke Neigung zum Braun-bezw. Rahnwerden zeigen, so ist bei diesen Weinen ein etwas stärkeres Einbrennen nach dem Abstich angezeigt.

Bei einem Rückblick auf das Jahr 1917 ergibt sich wieder für unsere Winzer die eindringliche Lehre, daß nur dort auf einen sicheren Erfolg gerechnet werden kann, wo es gelingt, die Vermehrung der Schädlinge durch Unterdrückung ihrer natürlichen Entwiskelungsbedingungen einzudämmen. Zweifellos wären auch in diesem Jahre die durch die Peronospora sowie die Lederbeerènkrankbeit verursachten Schäden wesentlich gemildert worden durch ein rechtzeitiges und vor allem sorgfältigeres Spritzen sowie durch eine bessere Bodenhearheitung der Weinberge. Immer noch findet die Tatsache noch zu wenig' Beachtung, daß der Infektion-herd der Peronospora auf der unteren Blattseite sich befindet, und daß die parasitischen Pilze nur dann ihr Gedeihen finden, wenn die Luft zwischen den Weinstöcken einen hohen Feuchtigkeitsgehalt aufweist. Es ist daher zur Herabminderung der Feuchtigkeit notwendig, die Luftbewegung in den Weinbergen zu fördern, was dureh ein rechtzeitiges Heften und Gipfeln, sowie lamentlich durch eine Beseitigung des Unkrautes erreicht werden kann.

Was die Düngung der Weinberge betrifft, so bat auch hier der Krieg zu einer Notlage geführt. Bei der sehr schwierigen und kostspieligen Beschaffung von Stallmist und bei der Knappheit der künstlichen Düngemittel kann als teilweiser Ersatz für die bisher benutzten Stickstoffdünger ein aus der Gewinnung von Weinsäure aus Weinhefe sich ergebendier Abfalldünger unseren Winzern empfohlen werden, der 1,5 bis $2,0 \%$ Stickstoff aufweist. 